\title{
Surveillance on Hand, Foot, and Mouth Disease in East Asia
}

\author{
Ta-Chien Chan*1, Chang-Chun Lee ${ }^{2}$ and Jing-Shiang Hwang ${ }^{3}$ \\ ${ }^{1}$ Research Center for Humanities and Social Sciences, Academia Sinica, Taipei City, Taiwan (Province of China); ${ }^{2}$ Genomics \\ Research Center, Academia Sinica, Taipei City, Taiwan (Province of China); ${ }^{3}$ Institute of Statistical Science, Academia Sinica, Taipei \\ City, Taiwan (Province of China)
}

\section{Objective}

Enterovirus epidemics, especially affecting young children, have occurred in South-East Asia every year. If the epidemic periods are inter-correlated among different areas, early warning signals could be issued to prevent or reduce the severity of the later epidemics in other areas. In this study, we integrated the available surveillance and weather data in East Asia to elucidate possible spatio-temporal correlations and weather conditions among different areas from low to high latitude.

\section{Introduction}

Regional disease surveillance as well as data transparency and sharing are the global trend for mitigating the threat of infectious diseases. The WHO has already played a leading role in FluNet (http:// www.who.int/influenza/gisrs_laboratory/flunet/en/ ) and DenguNet (http://www.who.int/csr/disease/dengue/denguenet/en/). However, the enterovirus-related infections which caused a high disease burden for pre-school children in South-East Asian regions over the last two decades still lack a comprehensive surveillance system in the region [1]. If the spreading pattern and a possible alert mechanism can be identified and set up, it will be beneficial for controlling hand, foot and mouth disease (HFMD) epidemics in East Asia. In some research findings, the transmission of HFMD was correlated with temperature, relative humidity, wind speed, precipitation, population density and the periods in which schools were open [2]. A delayed temporal trend was also found with the increase in latitude [3,4] . In this study, we tried to apply publicly available weekly surveillance data in Japan, Taiwan and Singapore to evaluate the spatio-temporal evolution of HFMD epidemics and how the weather conditions affect the HFMD epidemics.

\section{Methods}

HFMD surveillance data are collected online from the National Institute of Infectious Diseases in Japan, Taiwan CDC and Ministry of Health in Singapore. The study period started from week 31 of 2012 to week 27 of 2014 . There were 54 spatial areas including 47 prefectures in Japan, six areas in Taiwan and one area in Singapore. Due to different statistical units of the surveillance in different countries, we standardized the HFMD statistics separately by 54 areas. The weather data were collected from the National Climatic Data Center (NCDC) of the National Oceanic and Atmospheric Administration (NOAA). The daily mean temperature (Fahrenheit), mean dew point (Fahrenheit), mean sea level pressure (mb), mean visibility (miles), mean wind speed (knots), precipitation amount (inches), and maximum temperature (Fahrenheit) were calculated as the average values for the corresponding weeks. There were 205 weather stations included in this study after excluding the stations located at over 1,000 meters altitude or the stations located on the ocean. Latitudes were calculated from the centroid of each area. We used stepwise linear regression and ArcGIS for the statistical model and visualization, respectively.

\section{Results}

There were a total of 101 weeks during the study period. The dew point and latitude were significantly positively correlated with the increase of standardized HFMD values. The mean wind speed and sea level pressure were significantly negatively correlated with the increase of standardized HFMD values. Overall, the areas at higher latitudes had later HFMD epidemics than those at lower latitudes.

\section{Conclusions}

The weather conditions and the geographic locations could play an important role in affecting HFMD epidemics. Regional integrated surveillance of HFMD in East Asia is needed for mitigating the disease risk.

\section{Keywords}

Enterovirus; Climate change; Communicable diseases surveillance; Cross-border surveillance

\section{Acknowledgments}

This research was supported by a grant from the Ministry of Science and Technology, Taiwan (MOST 103-2621-M-001 -002).

\section{References}

1. Sabanathan S, Tan le V, Thwaites L, Wills B, Qui PT, et al. (2014) Enterovirus 71 related severe hand, foot and mouth disease outbreaks in South-East Asia: current situation and ongoing challenges. J Epidemiol Community Health 68: 500-502.

2. Wang Y, Feng Z, Yang Y (2012) Hand, Foot, and Mouth Disease in China: Patterns of Spread and Transmissibility (vol 22, pg 781, 2011). Epidemiology 23: 358-358.

3. Chang ZR, Zhang J, Sun JL, Zhang WD, Wang ZJ (2011) [Epidemiological features of hand, foot and mouth disease in China, 2008 - 2009]. Zhonghua Liu Xing Bing Xue Za Zhi 32: 676-680.

4. Chan TC, Hwang JS, Chen RH, King CC, Chiang PH (2014) Spatiotemporal analysis on enterovirus cases through integrated surveillance in Taiwan. BMC Public Health 14.

\footnotetext{
*Ta-Chien Chan

E-mail: dachianpig@gmail.com
} 